\title{
Serotonergic Modulation of Prefrontal Cortex during Negative Feedback in Probabilistic Reversal Learning
}

\author{
Elizabeth AT Evers', Roshan Cools*,2,3, Luke Clark ${ }^{3}$, Frederik M van der Veen', Jelle Jolles', \\ Barbara J Sahakian ${ }^{4}$ and Trevor W Robbins ${ }^{3}$ \\ 'Brain and Behavior Institute, University of Maastricht, Maastricht, The Netherlands; 'Department of Psychology, University of California, \\ Berkeley, CA, USA; ${ }^{3}$ Department of Experimental Psychology, University of Cambridge, Cambridge, UK; ${ }^{4}$ Department of Psychiatry, \\ Addenbrooke's Hospital, Cambridge, UK
}

\begin{abstract}
This study used functional magnetic resonance imaging to examine the effects of acute tryptophan (TRP) depletion (ATD), a wellrecognized method for inducing transient cerebral serotonin depletion, on brain activity during probabilistic reversal learning. Twelve healthy male volunteers received a TRP-depleting drink or a balanced amino-acid drink (placebo) in a double-blind crossover design. At $5 \mathrm{~h}$ after drink ingestion, subjects were scanned while performing a probabilistic reversal learning task and while viewing a flashing checkerboard. The probabilistic reversal learning task enabled the separate examination of the effects of ATD on behavioral reversal following negative feedback and negative feedback per se that was not followed by behavioral adaptation. Consistent with previous findings, behavioral reversal was accompanied by significant signal change in the right ventrolateral prefrontal cortex (PFC) and the dorsomedial prefrontal cortex. ATD enhanced reversal-related signal change in the dorsomedial PFC, but did not modulate the ventrolateral PFC response. The ATD-induced signal change in the dorsomedial PFC during behavioral reversal learning extended to trials where subjects received negative feedback but did not change their behavior. These data suggest that ATD affects reversal learning and the processing of aversive signals by modulation of the dorsomedial PFC.

Neuropsychopharmacology (2005) 30, I | 38-| | 47, advance online publication, 2 February 2005; doi: | 0.1038/sj.npp. I300663
\end{abstract}

Keywords: serotonin; reversal learning; functional imaging; punishment; inhibitory control; prefrontal cortex

\section{INTRODUCTION}

Serotonin (5-HT) has been extensively implicated in depressed mood and the processing of motivational signals (Graeff et al, 1986; Soubrie, 1986; Deakin, 1991; Wilkinson et al, 1995). Animal research has demonstrated that 5-HTenhancing drugs attenuate the aversive effects of brain stimulation (Patkina and Lapin, 1976; Graeff et al, 1986; Smith and Kennedy, 2003), and, conversely, potentiate selfstimulation in so-called 'reward' centers and enhance the motivational properties of stimuli predictive of rewards (Redgrave and Horrell, 1976; Aronson et al, 1995; SasakiAdams and Kelley, 2001; Orosco et al, 2004). Reduced motivation (anhedonia, apathy) is a cardinal feature of depression, where neuropsychological studies have further emphasized the relevance of incentive motivation and the processing of reinforcement; depression has been associated with a 'catastrophic response to perceived failure' (Beats et al, 1996) or an oversensitivity to negative feedback

\footnotetext{
*Correspondence: Dr R Cools, Helen Wills Neuroscience Institute, University of California, 132 Barker Hall, Berkeley, USA, Tel: + I 510 642 2839, E-mail: roshanco@berkeley.edu

Received 21 June 2004; revised 19 November 2004; accepted 29 November 2004

Online publication: I December 2004 at http://www.acnp.org/citations/ Npp I2060404027I/default.pdf
}

(Elliott et al, 1997; Steffens et al, 2001; Murphy et al, 2003). Selective 5-HT reuptake inhibitors exert antidepressant effects and acute reduction of central 5-HT function through dietary depletion of tryptophan (TRP), a precursor of 5-HT, can induce temporary depressive relapse in remitted patients (Young et al, 1985; Smith et al, 1997).

5-HT neurotransmission has been implicated not only in the processing of reward and punishment signals, but also in the inhibitory control of behavior (Soubrie, 1986; Evenden, 1999), where impulsive pathology is typically associated with reductions in central 5-HT (Coccaro et al, 1989; Cherek and Lane, 2000). Findings from studies with clinical populations are corroborated by animal studies linking impulsive choice in delay-discounting paradigms and premature responding in choice reaction-time tasks with 5-HT dysregulation (Harrison et al, 1997; Puumala and Sirvio, 1998; Koskinen et al, 2000; Mobini et al, 2000; Dalley et al, 2002; Liu et al, 2004).

These studies with animals have implicated particularly the medial and orbital prefrontal cortex (PFC) in impulsive performance (Dalley et al, 2002; Chudasama and Robbins, 2003; Liu et al, 2004), and this concurs indirectly with findings that manipulation of the 5-HT system in humans affects tasks that implicate the ventral and medial aspects of PFC (Robbins, 2000). Thus, neuropsychological studies have shown that acute TRP depletion (ATD) impairs 
performance on tasks of reversal learning, response inhibition, and affective decision-making (Park et al, 1994; Murphy et al, 2002; Walderhaug et al, 2002; Rogers et al, 2003), which have all been associated with ventral and/or medial PFC circuitry (Iversen and Mishkin, 1970; Jones and Mishkin, 1972; Dias et al, 1996; Rogers et al, 1999; O'Doherty et al, 2001; Cools et al, 2002; Fellows and Farah, 2003; Kringelbach and Rolls, 2003; Hornak et al, 2004).

While the ascending 5-HT projection has a widespread cortical distribution, receptor subtypes including the 5$\mathrm{HT}_{2 \mathrm{~A}}$ receptor show regional specificity to the frontal cortex and are overly expressed in medial and orbital regions in animal models of depression and anxiety (Poeggel et al, 2003; Preece et al, 2004). Structural and functional imaging studies in depressed patients also indicate reasonably selective abnormalities in the ventral and medial aspects of PFC (Drevets et al, 1997; Mayberg et al, 1999; Elliott et al, 2002; Ballmaier et al, 2004; Lacerda et al, 2004).

In the present study, we examined the effects of ATD on the blood oxygenation level-dependent (BOLD) response during probabilistic reversal learning, which requires the adaptation of behavior following changes in reward (and punishment) values as well as the maintenance of behavior in the face of misleading negative (probabilistic) feedback. ATD is a well-recognized research method for reducing central 5-HT in humans and studying the effects of low 5HT on cognition. ATD produces a rapid decrease in the synthesis and release of brain 5-HT (Nishizawa et al, 1997; Carpenter et al, 1998; Williams et al, 1999). TRP is depleted by ingesting an amino-acid mixture that does not contain TRP but does include other large neutral amino acids (LNAA) (Young et al, 1985). ATD is achieved by increasing protein synthesis in the liver with subsequent decreases in plasma TRP stores. In addition, the amino-acid load results in competition for the active transport system that the amino acids share for entry across the blood-brain barrier, resulting in reduced availability of TRP in the brain. The probabilistic reversal learning task enables the relatively separate examination of behavioral adaptation following negative feedback (aversive signals) and the processing of negative feedback without subsequent behavioral adaptation. We used the same probabilistic reversal learning task that was previously employed by Cools et al (2002). This study revealed significant BOLD changes during probabilistic reversal learning in the ventrolateral prefrontal cortex (VLPFC) and the dorsomedial PFC. Based on the strong $a$ priori association between depression, 5-HT, reversal learning, and orbital PFC, we decided not to restrict our regions of interest (ROIs) to the task-related brain areas but to extend these to the other orbital frontal regions not activated by the task. We predicted that ATD would modulate signal change in the ventral (including orbital) and medial PFC during the reception of negative feedback and the subsequent adaptation of behavior to the new contingencies.

\section{MATERIALS AND METHODS}

\section{Participants}

Twelve healthy right-handed male volunteers (18-28 years old; mean age of $23.8 \pm 2.8$ ) participated in this experiment.
The study was approved by the Local Research Ethical Committee in Cambridge and carried out in accordance with the Declaration of Helsinki. Participants were recruited via local advertisements, and screened for psychiatric and neurological disorders and MRI contraindications by means of prescreening questionnaires and interview by EATE. All volunteers gave written informed consent, and were paid for participation. The exclusion criteria were any history of cardiac, hepatic, renal, pulmonary, neurological or gastrointestinal disorder, medication use, and a history of major depression or bipolar affective disorder.

One participant vomited after ingesting the amino-acid mixture and was replaced by a substitute. One participant was excluded from the analysis due to poor performance on the reversal learning task (final $n=11$ ). His mean reaction time (RT) and the total number of trials on the reversal learning task were between 2.5 and 3.0 standard deviations higher than the group mean after the balanced drink.

\section{Experimental Design}

Participants attended two test sessions at least 1 week apart, and were administered either a TRP-depleted (TRP-) drink or a balanced (BAL) amino-acid drink in a double-blind crossover design (four participants received TRP- and seven received the BAL drink on the first session). Prior to a test session, volunteers fasted overnight and low-protein food was provided during the test days. Following a resting period of $5 \mathrm{~h}(4.5 \mathrm{~h}, \mathrm{SD}=35 \mathrm{~min}$, in the TRP - condition and $5.0 \mathrm{~h}, \mathrm{SD}=40 \mathrm{~min}$, in the balanced condition), to ensure stable and low TRP levels (Riedel et al, 1999), participants entered the functional magnetic resonance imaging (fMRI) scanner at the Wolfson Brain Imaging Centre (WBIC). They were scanned while performing three blocks of the probabilistic reversal learning task each for about 9 min (Cools et al, 2002) and the checkerboard task. Behavioral performance on the reversal learning task was assessed using button presses on a response box. Structural scans were obtained at the end of a test session or on a separate session.

\section{Probabilistic Reversal Learning Task}

The probabilistic reversal learning task was described in detail by Cools et al (2002). The task is a two-choice visual discrimination task where the same two abstract patterns were presented on each trial. Using trial-and-error feedback after each response (a green happy face or a red sad face), subjects learned to select the stimulus that was usually correct. This rule intermittently reversed so that the other stimulus was usually correct. Consequently, responding had to be adjusted in order to gain reward and avoid punishment. On a minority of trials (10-20\%) false-negative feedback was provided to a correct response, the so-called 'probabilistic errors' (0-4 per reversal). Reversal of the stimulus-reward contingency occurred after 10-15 correct responses (including probabilistic errors). Participants performed three successive 9-min blocks of the task, each taking 140-160 trials (block length was determined by the number of errors made). Stimuli were presented for a $2000 \mathrm{~ms}$ response window (RTs $>2000 \mathrm{~ms}$ were followed by a 'too late' message). Feedback was presented immediately 
after the response for $500 \mathrm{~ms}$. After feedback, the stimuli were replaced by a fixation cross for a variable duration so that the overall interstimulus interval was $3215 \mathrm{~ms}$, enabling precise desynchronization from the repetition time (TR) of $1600 \mathrm{~ms}$.

Four types of events were modeled: (i) a correct response followed by positive feedback, (ii) a correct response followed by negative feedback (probabilistic error), (iii) an incorrect response where the subject reversed on the subsequent trial (reversal switch error), and (iv) an incorrect response where the subject did not reverse (ie perseverated) on the subsequent trial (preceding error). Spontaneous discrimination errors (those which could not be categorized as reversal or probabilistic errors) were not included in the model.

\section{Checkerboard Task}

The checkerboard task was a passive visual task where the subject viewed two configurations of black and white squares in an $8 \times 8$ matrix that switched at a frequency of $8 \mathrm{~Hz}$. Using a blocked $\mathrm{ABAB}$ design, $20 \mathrm{~s}$ checkerboard blocks alternated with $20 \mathrm{~s}$ crosshair fixation for six cycles, taking a total of $4 \mathrm{~min}$.

\section{Amino-Acid Mixture}

The TRP-deficient amino-acid drink (TRP-) contained a total of $75 \mathrm{~g}$ of amino acids using the proportions described by Young et al (1985): $4.1 \mathrm{~g}$ L-alanine, $2.4 \mathrm{~g}$ glycine, $2.4 \mathrm{~g}$ $\mathrm{L}$-histidine, $6.0 \mathrm{~g}$ L-isoleucine, $10.1 \mathrm{~g}$ L-leucine, $6.7 \mathrm{~g} \mathrm{~L}$-lysine, $4.3 \mathrm{~g}$ L-phenylalanine, $9.2 \mathrm{~g}$ L-proline, $5.2 \mathrm{~g}$ L-serine, $4.3 \mathrm{~g}$ L-threonine, $5.2 \mathrm{~g}$ L-tyrosine, $6.7 \mathrm{~g}$ L-valine, $3.7 \mathrm{~g}$ L-arginine, $2.0 \mathrm{~g}$ L-cysteine, and $3.0 \mathrm{~g}$ L-methionine (SHS International Ltd, Liverpool, UK). The balanced mixture contained the same amino acids, plus $3.0 \mathrm{~g}$ TRP. The drinks were prepared with $200 \mathrm{ml}$ tap water and fruit flavoring to compensate for the unpleasant taste.

\section{Biochemical Measures}

Blood samples $(10 \mathrm{ml})$ were taken prior to ingestion of the amino-acid mixture and after the fMRI scan (about $6.5 \mathrm{~h}$ later), to determine the plasma TRP level and the TRP/ $\Sigma$ LNAA ratio. This ratio is important because the uptake of TRP in the brain is strongly associated with the amounts of other LNAA competing at the blood-brain barrier. Venous samples were taken in lithium heparin tubes, centrifuged, and stored at $-20^{\circ} \mathrm{C}$. Plasma TRP was determined by an isocratic high-performance liquid chromatography (HPLC) method of analysis. Plasma proteins were removed by precipitation with $3 \%$ trichloroacetic acid (TCA) and centrifugation at 3000 revs, $4^{\circ}$ for $10 \mathrm{~min}$, and then pipetted into heparin aliquots. An aliquot was then diluted in mobile phase before injection into the HPLC analysis column. Fluorescence end-point detection was used to identify TRP.

Paired-sample $t$-tests were used to compare the two baseline measurements of plasma TRP levels and TRP/ $\Sigma$ LNAA ratios, and to compare measurements of plasma TRP levels and the TRP/ $\Sigma$ LNAA ratio in the balanced and TRP- condition. A repeated-measures ANOVA was performed to look at the effect of ATD on plasma TRP levels and the TRP/ $\Sigma$ LNAA ratios.

\section{Psychological Ratings}

Visual Analogue Scales (VAS) containing the items drowsy, sad, happy, anxious and nauseous were administered five times during the test day (at roughly $90 \mathrm{~min}$ intervals). The Positive and Negative Affect Scale (PANAS; Watson et al, 1988) was completed prior to ingestion and after the scan. Repeated-measures ANOVA with drink treatment (TRP - and balanced) and time (two time points for the VAS, and two for the PANAS). Greenhouse-Geisser corrections were applied when the sphericity assumption was violated.

\section{Behavioral Data Analysis}

Dependent measures were the number of reversal contingencies during the task as a whole, the number of errors due to switching after a probabilistic error, mean RT, and a maintenance score that was calculated by dividing the number of errors made following five correct responses but prior to the next contingency reversal by the number of trials remaining prior to the next contingency reversal (adapted from Swainson et al, 2000) for each reversal block. Data were analyzed using repeated-measures ANOVA with block (1-3) and treatment (balanced and TRP-) as withinsubjects factors and order of drug treatment (balanced first or TRP - first) as between-subjects factor. Greenhouse-Geisser corrections were applied when the sphericity assumption was violated. Simple effects of block and treatment were analyzed using post hoc tests with Bonferroni correction for multiple comparisons. Medians were used for analysis because RTs were not normally distributed. Measures that were not normally distributed were analyzed with the nonparametric Wilcoxon signed ranks test.

\section{Image Acquisition}

Participants were scanned in a $3 \mathrm{~T}$ Bruker Medspec scanner (S300; Bruker, Ettlingen, Germany), at the WBIC. T2*weighted gradient echo planner images (EPI) (TE $27 \mathrm{~ms}$ ) were acquired with blood oxygenation level-dependent (BOLD) contrasts. A whole-brain acquisition consisted of 21 slices (TR $1.6 \mathrm{~s}$; voxel size before normalization $1.56 \times 1.56 \times 5 \mathrm{~mm}^{3}$ and after normalization $3 \times 3 \times 3 \mathrm{~mm}^{3}$; inter-slice gap $1 \mathrm{~mm}$; matrix size $128 \times 128$; bandwidth $100 \mathrm{kHz}$; oblique orientation) and the total number of volumes acquired varied from run to run (from 142 to 166) depending on the participant's performance. In addition, high-resolution T1-weighted images for spatial normalization were acquired of each participant (voxel size $1 \times 1 \times 1 \mathrm{~mm}^{3}$ ). We were unable to acquire reliable data from a section of ventromedial PFC because of susceptibility artifacts.

\section{Image Analysis}

Data analysis was performed using SPM99 and SPM2 (Statistical Parametric Mapping; Wellcome Department 
of Cognitive Neurology, London, UK). Preprocessing procedures consisted of (linear) slice acquisition time correction, within-subject realignment (SPM2), geometric undistortion using fieldmaps (Cusack et al, 2003), spatial normalization using each individual subject's skullstripped SPGR (using the Brain Extraction Tool; Smith, 2002), and the Montreal Neurological Institute (MNI) skull-stripped structural template (SPM2) and spatial smoothing using a Gaussian kernel $(10 \mathrm{~mm}$ full-width at half-maximum).

A canonical hemodynamic response was used as a covariate in a general linear model and a parameter estimate was generated for each voxel for each event type. For each event, the hemodynamic response function was modeled to the onset of the response, which co-occurred with the presentation of the feedback for the reversal learning task.

For each subject, the following contrasts were computed: (i) Main task effect 1: Reversal switch errors $v s$ baseline correct responses for the balanced condition only. (ii) Main task effect 2: Reversal nonswitch errors (which included probabilistic and preceding errors which were not followed by the subject switching responding) vs baseline correct responses for the balanced condition only. (iii) Main task effect 3: Reversal switch errors vs the other nonswitch errors for the balanced condition only. (iv) Treatment $\times$ task interaction 1 , reflecting the effect of ATD on task effect 1. (v) Treatment $\times$ task interaction 2, reflecting the effect of ATD on task effect 2. (vi) Treatment $\times$ task interaction 3, reflecting the effect of ATD on task effect 3. Thus, treatment was modeled as a within-subject variable within each individual's general linear model. For the checkerboard task, an epoch (box-car) design contrasted checkerboard visual stimulation with crosshair fixation. Individual contrast images were taken to a second level analysis in which $t$-values were calculated for each voxel, treating inter-subject variability as a random effect.

The MarsBar tool (Brett et al, 2002) was used to average signal within independently defined ROIs at the group level. ROIs for the reversal task analysis were defined from the activation peaks found by Cools et al (2002); $10 \mathrm{~mm}$ spheres (corresponding to the smoothing filter) were built around the dorsomedial PFC $(x, y, z=8,32,52)$, right $\operatorname{VLPFC}(x, y$, $z=38,24,-2)$ and left VLPFC $(x, y, z=-32,24,-4)$. The random effects model was then reapplied to the average signal within these ROIs to test the statistical significance of the contrasts of interest (a one-sample $t$-test). Average signal change was extracted from each ROI and these are the values reported in Figure 2. In addition, we also performed whole-brain analyses. Both ROI and whole-brain analyses were thresholded at $P<0.05$ (corrected for multiple comparisons). Given the a priori prediction concerning the modulation of the orbitofrontal cortex by ATD during reversal learning, we also examined the inferior, medial, and superior orbitofrontal cortex using ROIs from the Automated Anatomical Labelling (AAL) map based on the MNI average brain (Tzourio-Mazoyer et al, 2002), also thresholded at $P<0.05$. Finally, the main effect of ATD was assessed by contrasting all task-related regressors from the TRP - condition with all task-related regressors from the balanced condition.

\section{RESULTS}

\section{Functional Imaging Data}

All significant task-related effects from the balanced condition are shown in Table 1. ATD significantly increased the BOLD response in the dorsomedial PFC during reversal switch errors relative to correct baseline responses. This effect reached significance in both the ROI (contrast iv; $\mathrm{T}_{10}=2.04 ; P=0.03$; Figure 1 ) and whole-brain analyses (Talairach coordinates $x, y, z=9,39,48 ; \mathrm{T}_{10}=11.95$; $\left.P_{\text {corrected }}=0.006\right)$. However, the effect of ATD did not reach

Table I Significant Task Effects Revealed by Regions of Interest Analyses (From the Balanced Condition Only)

\begin{tabular}{lcc}
\hline Increased activation & T-values & $P$ \\
\hline Reversal switch errors minus correct responses (contrast i) & \\
Dorsomedial PFC & 4.22 & $<0.001$ \\
Right ventrolateral PFC & 7.02 & $<0.001$ \\
Left ventrolateral PFC & 3.43 & $<0.01$ \\
& & \\
Other non-switch errors minus correct responses (contrast ii) & $<0.001$ \\
Right ventrolateral PFC & 4.83 & $<0.01$ \\
Left ventrolateral PFC & 3.16 & \\
& & $<0.001$ \\
Reversal switch errors minus other nonswitch errors (contrast iii) & $<0.001$ \\
Dorsomedial PFC & 5.15 & \\
Right ventrolateral PFC & 4.55 & \\
\hline
\end{tabular}

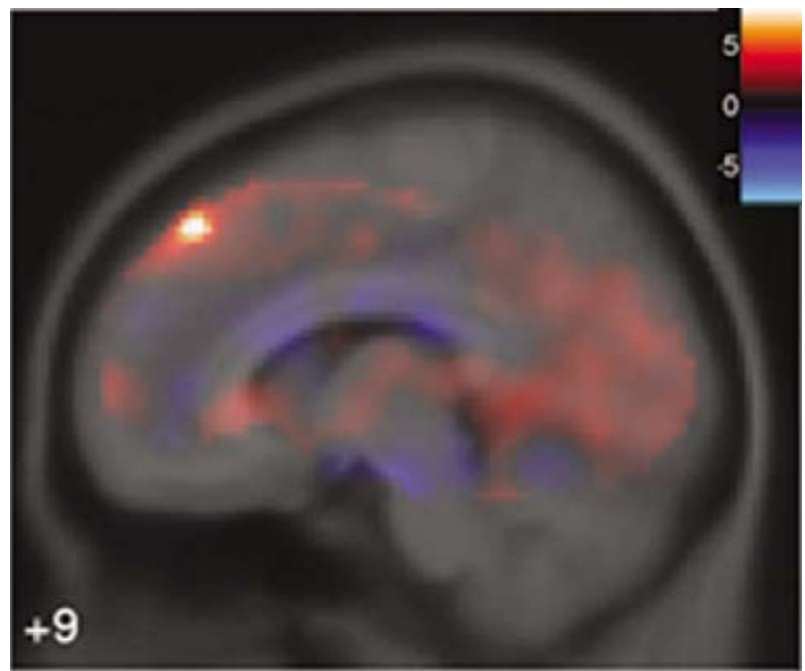

Figure I The continuous $t$-value image (statistical parametric map) (color-coding according to legend in top-right corner: light blue = extreme negative $t$-value and light red =extreme positive $t$-value) is shown as a sagittal section ( $\mathrm{MNI}$ coördinate $y=9$ ) superimposed upon the average $\mathrm{MNI} \mathrm{TI}$ template (average of I 52 brains). The $t$-map represents functional activation changes following ATD relative to the balanced (placebo) condition during reversal switch errors relative to baseline correct responses (a treatment $\times$ task interaction effect). Whole-brain (and $\mathrm{ROI}$ ) analyses revealed a significant signal increase following TRP depletion in the dorsomedial PFC, centered on $M N I$ coördinates $(x, y, z)=(9,39,48)$ $\left(T_{10}=11.95, P_{\text {corrected }}=0.006\right)$. 

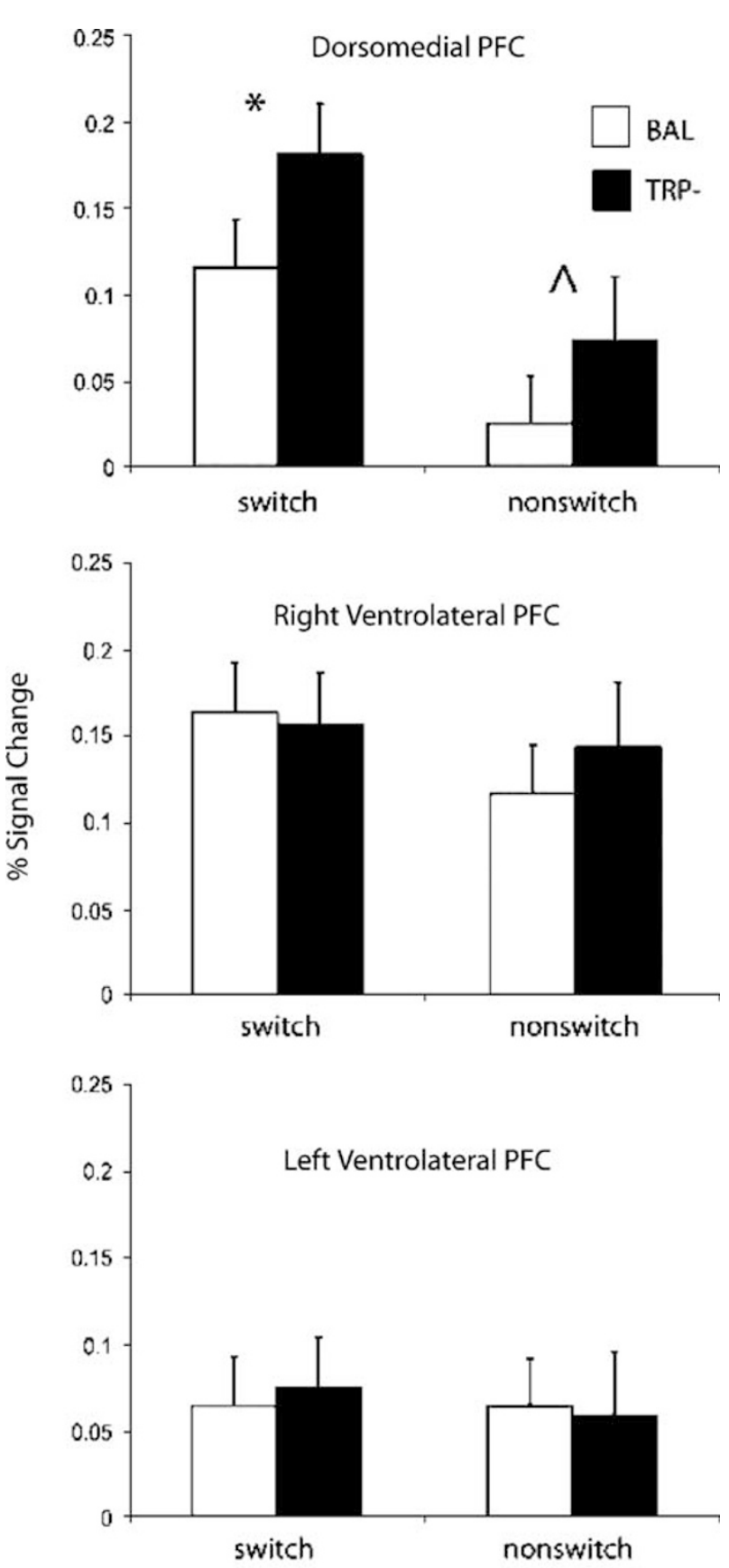

Figure 2 Percent signal change is shown for three ROI: the dorsomedial PFC, the right and the left ventrolateral PFC for reversal switch errors (relative to baseline correct responses), and nonswitch errors (including probabilistic and preceding errors relative to baseline correct responses) in the BAL and TRP - conditions. Errors bars represent SEMs. ROI (spheres with $10 \mathrm{~mm}$ radius) were defined from peak coordinates from Cools et al (2002) (see Materials and methods section for coordinates). $* P<0.05$, $\wedge P<0.1$.

significance when the reversal switch errors were compared with the other nonswitch errors (contrast vi; $\mathrm{T}_{10}=1.33$; $P=0.1)$. Furthermore, the increase in signal in the dorsomedial PFC tended towards significance when the reversal nonswitch errors were compared with baseline correct responses (contrast v; $\mathrm{T}_{10}=1.44 ; \quad P=0.09$ ) (Figure 2). Thus, ATD increased signal changes during all negative feedback, irrespective of whether the errors were followed by behavioral reversal. ATD did not affect the BOLD response in the left and right VLPFC $\left(\mathrm{T}_{10}=-0.13\right.$, $P=0.55$ and $\mathrm{T}_{10}=-0.21, P=0.6$, respectively). Furthermore, ATD did not significantly affect global activation changes during the task, as revealed by both whole-brain and ROI analyses (ROI analyses: left VLPFC: $P=0.33$; right VLPFC: $P=0.32$; medial PFC: $P=0.23$ ).

While an ATD-induced increase in the orbitofrontal cortex during the reversal switch errors compared with correct responses did not reach significance in the ROI or whole-brain analysis according to our criterion (AAL's left middle orbital gyrus: $\mathrm{T}_{10}=1.12 ; P=0.14$ ), for completion we report that whole-brain analysis revealed a nonsignificant effect at $x, y, z=-48,42,-3\left(\mathrm{~T}_{10}=5.72 ; Z=3.7\right)$.

Supplementary analysis revealed that our findings are not confounded by the fact that four participants started with the TRP - condition and seven participants started with the balanced condition. This analysis of the individual parameter estimates, extracted from the dorsomedial PFC ROI (reversal switch errors minus baseline correct responses), revealed that the effect of ATD was not qualified by testing order $\left(\right.$ ATD $\times$ testing order interaction: $\mathrm{F}_{1,9}=0.5, P=0.5$ ). No differences in signal change were observed between subjects who ingested the TRP - drink on the first occasion (mean signal change $=0.16$ ) and subjects who ingested the TRP - drink on the second occasion (mean signal change $=0.19$ ), and no differences were observed between subjects who ingested the balanced mixture on the first occasion (mean signal change $=0.11$ ) and subjects who ingested the balanced mixture on the second occasion (mean signal change $=0.12$ ).

Whole-brain analyses did not reveal any other significant effects.

\section{Behavioral Effects of ATD on Reversal Learning}

There was a nonsignificant tendency for ATD to slow overall RT $\left(\mathrm{F}_{1,9}=4.72, P=0.06\right)$; mean $\mathrm{RT}$, TRP $-=554 \mathrm{~ms}$, $\mathrm{BAL}=522 \mathrm{~ms})$. No other differences were found between the TRP - and balanced condition. Mean values are presented in Table 2.

\section{Checkerboard Task}

Visual stimulation was associated with a large and highly significant activation cluster $(T=13.63, P=0.002)$ in the occipital cortex. No effects of ATD on visual stimulation in

Table 2 Behavioral Effects of ATD

\begin{tabular}{lccc}
\hline & Balanced & TRP - & P-value \\
\hline Total number of reversals & $26.8(0.1)$ & $26.1(0.5)$ & 0.26 \\
$\begin{array}{l}\text { Total number of switches after } \\
\text { probabilistic errors }\end{array}$ & $10.3(2.8)$ & $10.3(2.9)$ & 0.55 \\
Mean maintenance score & $0.06(0.0)$ & $0.06(0.1)$ & 0.64 \\
Mean overall RT & $523(19)$ & $554(31)^{\mathrm{a}}$ & 0.06 \\
\hline
\end{tabular}

Values represent means (SEMs).

a The effect of ATD on mean RT tended towards significance at $P=0.06$. 
Table 3 Psychological Ratings of Mood at Baseline t0 and PostScanning t6.5

\begin{tabular}{lccccc}
\hline & BAL t0 & BAL t6.5 & TRP- to & TRP-t6.5 & P-value \\
\hline VAS & & & & & \\
Drowsy & $2.0(2.1)$ & $3.1(2.3)$ & $2.1(1.8)$ & $3.4(3.1)$ & 0.07 \\
Sad & $1.0(0.7)$ & $1.8(1.6)$ & $1.8(1.6)$ & $2.0(1.6)$ & 0.60 \\
Happy & $5.2(2.0)$ & $4.0(2.3)$ & $5.5(1.5)$ & $5.0(1.4)$ & 0.68 \\
Anxious & $2.6(1.8)$ & $1.2(1.5)$ & $1.9(1.4)$ & $1.4(1.0)$ & 0.37 \\
Nausea & $1.6(2.0)$ & $1.4(1.1)$ & $1.9(2.0)$ & $1.7(1.3)$ & 0.56 \\
& & & & & \\
PANAS & & & & & \\
Positive & $26.9(1.9)$ & $23.7(1.6)$ & $28.6(0.9)$ & $23.6(1.8)$ & 0.36 \\
Negative & $13.4(0.8)$ & $11.8(0.5)$ & $13.4(0.7)$ & $13.3(0.8)$ & 0.14 \\
\hline
\end{tabular}

$\mathrm{BAL}=$ balanced condition; TRP $-=$ tryptophan-depleted condition.

this region in occipital cortex were found, even at $P=0.01$ uncorrected for whole-brain volume.

\section{Biochemical Measures}

The analysis of amino-acid levels revealed that the depletion was successful. No differences between conditions were found in terms of plasma TRP level (mean/SEM: BAL 12.2/ 2.3; TRP 12.3/3) and ratio TRP/ 2 LNAA's (BAL 0.19/0.02; TRP $-0.16 / 0.02$ ) at baseline. After $6.5 \mathrm{~h}$, plasma TRP and the ratio TRP/ $\Sigma$ LNAA were significantly lower in the TRPcondition compared with the balanced condition (TRP levels: $\mathrm{BAL} 18.2 / 6.3 ; \mathrm{TRP}-4.4 / 1.8 ; T_{10}=8.3, P<1$ and ratios: $\mathrm{BAL} 0.16 / 0.03$; TRP $-0.04 / 0.01 ; T_{10}=4.0, P<0.01$, respectively). Following the TRP-drink, plasma TRP was reduced by $64 \%$ and the ratio TRP/ $\Sigma$ LNAA's by $74 \%$ relative to baseline $\mathrm{t} 0$. Following the balanced drink, the plasma TRP was increased by $50 \%$ and the ratio TRP/ $\Sigma$ LNAA was reduced by $16 \%$.

\section{Psychological Ratings}

No interaction effects of ATD by time on PANAS-positive and -negative effect scores were observed (positive effect: $\mathrm{F}_{1,10}=1.1, P=0.3$; negative effect: $\mathrm{F}_{1,10}=1.0, P=0.3$ ), while there were significant main effects of time across drink treatment on both scores (positive effect: $\mathrm{F}_{1,10}=6.7$, $P=0.03$; negative effect: $\mathrm{F}_{1,10}=6.1, P=0.03$ ) (Table 3 ). Similarly, while some main effects of time reached significance for VAS scores (Drowsiness increased over time: $\mathrm{F}_{1,10}=14.7, P=0.003$; Happiness and anxiety decreased over time: $\mathrm{F}_{1,10}=011.3, P=0.007$ and $\mathrm{F}_{1,10}=11.1$, $P=0.008$, respectively), no significant interaction effects between time and drink treatment on VAS scores were observed (Drowsiness: $\mathrm{F}_{1,10}=0.04, \quad P=0.8$; Sadness: $\mathrm{F}_{1,10}=0.7, P=0.4$; Happiness: $\mathrm{F}_{1,10}=1.0, P=0.3$; Anxiety: $\mathrm{F}_{1,10}=1.6, P=0.2$; Nausea: $\mathrm{F}_{1,10}=0.03, P=0.9$ ).

\section{DISCUSSION}

The present findings reveal that acute depletion of central 5HT in young healthy male volunteers significantly increased the task-related BOLD response in the dorsomedial PFC during probabilistic reversal learning. By contrast, ATD did not modulate task-related signal change in the ventrolateral PFC. These effects contrast with those observed following manipulation of the central dopamine system in young healthy volunteers, which reduced the BOLD signal in the left ventrolateral PFC during reversal learning but did not affect signal in the dorsomedial PFC (Clark et al, 2004), and suggest that changes in the 5-HT system affect probabilistic reversal learning via action in the dorsomedial PFC.

The observed reduction in plasma TRP was comparable to that seen in previous studies (Young et al, 1985; Murphy et al, 2002) and data from both animal and human studies indicate that acute precursor depletion induces significant reductions in the synthesis and release of brain 5-HT (Biggio et al, 1974; Nishizawa et al, 1997; Carpenter et al, 1998). The BOLD changes following ATD are unlikely to reflect indirect effects of the dietary manipulation on mood or arousal, because no effects of ATD on any of the subjective ratings were observed. As predicted, ATD did not affect depressive symptomatology in this sample of young volunteers without a history of psychiatric disorder (Benkelfat et al, 1994). Although there was a tendency towards an overall slowing of response latencies following depletion of central 5-HT, the BOLD changes were not accompanied by significant behavioral changes and are thus unlikely to reflect increased task difficulty. The observed tendency towards a behavioral effect concurs with previous observations that ATD induces significantly slower latencies in a neuropsychological version of the probabilistic reversal learning task (Murphy et al, 2002), which involved only a single reversal of contingencies. Murphy et al (2002) showed that ATD slowed responding only in a first test session, in which the task was novel to the subjects. In light of these previous observations, we suggest that the present behavioral measure of repeated, well-practiced and serial reversal learning was not sufficiently sensitive to reveal significant behavioral effects of ATD. Furthermore, we propose that the physiological BOLD response provided a more sensitive measure of the effects of ATD, hypothetically accompanying behavioral changes that surface only in more sensitive, single reversal learning paradigms.

The increased response in the dorsomedial PFC after 5-HT depletion was significant when the reversal switch errors were compared with correct responses, but did not reach significance when the reversal switch errors were compared with the reversal nonswitch errors. Moreover, ATD increased (although only marginally significantly) the BOLD signal during the reversal nonswitch errors relative to correct responses. The ATD effect was therefore not restricted to errors that were followed by a behavioral switch, but extended to the other error types where negative feedback was presented without subsequent reversal. The present task thus implicates 5 -HT in processes other than reversal switching, which may include the detection/ monitoring of errors and/or conflict. This is consistent with cognitive models of medial frontal cortex function, which emphasize the region's involvement in error detection and conflict monitoring (Paus et al, 1993; Carter et al, 1998; Gehring and Fencsik, 2001; Kerns et al, 2004; MacDonald et al, 2000; Garavan et al, 2003). 
Based on evidence for a role of 5-HT in the processing of aversive signals, as well as models of depression as an oversensitivity to negative feedback (Elliott et al, 1997; Steffens et al, 2001; Murphy et al, 2003; Graeff et al, 1986; Smith and Kennedy, 2003), we hypothesize that the increased BOLD signal in the dorsomedial PFC reflects enhanced processing of aversive signals (error feedback) leading to enhanced response conflict on both switch and nonswitch trials. Clearly, the task does not fully dissociate negative feedback, conflict and behavioral switching: negative feedback initiates conflict and subsequent reversal, and a probabilistic task places demands on inhibitory control to maintain responding after misleading feedback. This is illustrated by the findings of Murphy et al (2003), showing that depressed people exhibit impaired maintenance of responding in the probabilistic reversal learning task, specifically following misleading negative feedback. Serotonergic modulation of medial frontal function is also consistent with the selective abnormalities seen in this region in structural imaging and post-mortem investigations in mood disorders (Drevets et al, 1997; Rubinsztein et al, 2001; Merali et al, 2004).

Altered 5-HT neurotransmission in the PFC has also been associated with failures of inhibitory control (Leyton et al, 2001; Clarke et al, 2004; Liu et al, 2004). A recent study has demonstrated increased perseverative responding in marmosets following prefrontal 5-HT depletion, using a simple (nonprobabilistic) reversal learning task (Clarke et al, 2004). There are significant methodological differences between the tasks used in that study and the current fMRI task. Primarily, the probabilistic nature of the fMRI task increases the incidence of error processing and is likely to enhance the salience of punishment during the task. This may render the task particularly sensitive to changes in punishment sensitivity. The nature of punishment is also different in the two tasks. The fMRI task employs a specific error signal (a red sad face), whereas reversal is indicated to the marmoset by the omission of reward for the previously relevant stimulus, co-occurring with unexpected reward for the newly relevant stimulus. The hypothesized increase in sensitivity to negative feedback induced by ATD in the current study may coexist with a deficit in the inhibitory control of behavior. In probabilistic reversal learning, this may diminish the ability to maintain responding to the relevant stimulus following misleading negative feedback, as shown in depressed patients by Murphy et al (2003). However, in a nonprobabilistic reversal learning task that de-emphasizes the processing of punishment cues, behavioral changes may surface as impaired inhibitory control, in the form of perseverative responding. Of course, it also remains to be established at the present time whether a global depletion of 5-HT precursor in humans is functionally equivalent to a prefrontal lesion of 5-HT fibres in marmosets.

The present pattern contrasts with effects of the (dopamine) DA D2 receptor antagonist as well as the indirect catecholamine agonist methylphenidate, which resulted in significant reductions in the BOLD signal in the ventrolateral PFC that were specific to behavioral switching and cannot have been solely induced by the reception of negative feedback (Clark et al, 2004). This dissociation concurs with the observation that the indolea- mine 5-HT modulates in particular a medial and orbital prefrontal neural system associated with associative learning and affective decision making, while catecholamines (ie DA and norepinephrine) affect predominantly a system connecting to the lateral PFC associated with set shifting, response inhibition, and working memory (Robbins, 2000; Aron et al, 2004).

The PFC is densely innervated with large ascending serotonergic projections from the raphé nuclei and there are several mechanisms via which 5-HT may modulate PFC neuronal responses. For example, evidence from in vitro studies show that activation of $5-\mathrm{HT}_{2 \mathrm{~A}}$ receptors induces a rapid increase in excitatory postsynaptic potentials/currents in virtually all layer $\mathrm{V}$ pyramidal cells of neocortex, but most prominently in medial PFC and other frontal regions where $5-\mathrm{HT}_{2 \mathrm{~A}}$ receptors are enriched (Marek and Aghajanian, 1998). Activation of postsynaptic $5-\mathrm{HT}_{1 \mathrm{~A}}$ receptors in the $\mathrm{PFC}$, on the other hand, have been found to inhibit pyramidal cell activity (Puig et al, 2004). It is unlikely that our findings reflect vasoconstrictive or vasodilative effects of ATD leading to indirect (non-neuronal) changes in the hemodynamic response, because the increase was regionally selective to dorsomedial PFC and did not extend to other task-related brain areas, which were modulated by dopaminergic drugs. Vascular effects would be predicted to affect task-related signal globally. Moreover, ATD did not affect significantly task-related signal during the presentation of a checkerboard task that elicited a robust response in visual striate cortex.

The present study only used male volunteers. Previous studies showed that females are more sensitive to mood effects after ATD than males (Ellenbogen et al, 1996) and thus male volunteers were selected for the present study to avoid the potentially confounding effects of mood on task performance and task-related BOLD signal change. However, the cognitive and biological effects of 5-HT depletion may be markedly greater in female subjects (Nishizawa et al, 1997; Harmer et al, 2003). For example, ATD reduced the rate of 5 -HT synthesis by 40 times in females compared to only 10 times in males (Nishizawa et al, 1997). Our results might be representative for a male population only, and need to be extended to female subjects in subsequent research. In a further feature of the experimental design, we employed a $75 \mathrm{~g}$ TRP - mixture rather than the more typical $100 \mathrm{~g}$ mixture, in order to minimize the incidence of nausea and vomiting during the scanning procedure. The biochemical analyses confirmed a successful depletion with this reduced volume, whereby plasma TRP was reduced by $64 \%$ on average, and the ratio of TRP to other LNAA was reduced by $74 \%$. The ratio of TRP/ $\Sigma$ LNAA was virtually unaffected by the balanced mixture (only a $16 \%$ decrease relative to baseline), and this ratio is associated most closely with reduced 5-HT synthesis.

In conclusion, acute depletion of brain 5-HT levels in healthy male volunteers increased the BOLD signal in dorsomedial PFC during performance of a task that involves feedback processing and the adaptation of behavior. The effects of 5-HT were related to the processing of negative feedback more than behavioral adaptation per se, and this provides one of the first direct demonstrations of 
serotonergic modulation of medial frontal processes in human subjects. During reversal learning, the dorsomedial PFC response is part of an extended network of frontal activity, and these data highlight the potential of pharmacological fMRI as a tool for dissecting the neurochemical contributions to cognitive control mechanisms.

\section{ACKNOWLEDGEMENTS}

We thank Wim Riedel and Ed Bullmore for helpful discussion, Matthew Brett for analysis support, Ruth Bisbrown-Chippendale and Clair Sleator from the Wolfson Brain Imaging Centre (Cambridge, UK) for radiographic assistance, and nursing staff of the Wellcome Trust Clinical Research Facility at Addenbrooke's hospital. This study was funded by a Wellcome Trust programme grant to TW Robbins, BJ Everitt, AC Roberts, and BJ Sahakian (LC), and completed within the MRC Centre for Behavioural and Clinical Neuroscience. EATE is funded by the ZonMW grant 912-02-050 from the dutch Organization for Scientific Research (NWO). RC holds a Royal Society Dorothy Hodgkin Fellowship and a Junior Research Fellowship from St John's College, Cambridge, UK.

\section{REFERENCES}

Aron AR, Robbins TW, Poldrack RA (2004). Inhibition and the right inferior frontal cortex. Trends Cogn Sci 8: 170-177.

Aronson SC, Black JE, McDougle CJ, Scanley BE, Jatlow P, Kosten TR et al (1995). Serotonergic mechanisms of cocaine effects in humans. Psychopharmacology (Berl) 119: 179-185.

Ballmaier M, Toga AW, Blanton RE, Sowell ER, Lavretsky H, Peterson J et al (2004). Anterior cingulate, gyrus rectus, and orbitofrontal abnormalities in elderly depressed patients: an MRI-based parcellation of the prefrontal cortex. Am J Psychiatry 161: 99-108.

Beats BC, Sahakian BJ, Levy R (1996). Cognitive performance in tests sensitive to frontal lobe dysfunction in the elderly depressed. Psychol Med 26: 591-603.

Benkelfat C, Ellenbogen MA, Dean P, Palmour RM, Young SN (1994). Mood-lowering effect of tryptophan depletion. Enhanced susceptibility in young men at genetic risk for major affective disorders. Arch Gen Psychiatry 51: 687-697.

Biggio G, Fadda F, Fanni P, Tagliamonte A, Gessa GL (1974). Rapid depletion of serum tryptophan, brain tryptophan, serotonin and 5-hydroxyindoleacetic acid by a tryptophan-free diet. Life Sci 14: 1321-1329.

Brett M, Anton J-L, Valabregue R, Poline J-B (2002). Region of interest analysis using an SPM toolbox [abstract]. NeuroImage 16: S497.

Carpenter LL, Anderson GM, Pelton GH, Gudin JA, Kirwin PD, Price LH et al (1998). Tryptophan depletion during continuous CSF sampling in healthy human subjects. Neuropsychopharmacology 19: 26-35.

Carter CS, Braver TS, Barch DM, Botvinick MM, Noll D, Cohen JD (1998). Anterior cingulate cortex, error detection, and the online monitoring of performance. Science 280: 747-749.

Cherek DR, Lane SD (2000). Fenfluramine effects on impulsivity in a sample of adults with and without history of conduct disorder. Psychopharmacology (Berl) 152: 149-156.

Chudasama Y, Robbins TW (2003). Dissociable contributions of the orbitofrontal and infralimbic cortex to pavlovian autoshaping and discrimination reversal learning: further evidence for the functional heterogeneity of the rodent frontal cortex. $J$ Neurosci 23: $8771-8780$.
Clark L, Cools R, Evers LE, van der Veen F, Jolles J, Sahakian BJ et al (2004). Neurochemical modulation of prefrontal cortex function. FENS Abstracts 2: A205.1.

Clarke HF, Dalley JW, Crofts HS, Robbins TW, Roberts AC (2004). Cognitive inflexibility after prefrontal serotonin depletion. Science 304: 878-880.

Coccaro EF, Siever LJ, Klar HM, Maurer G, Cochrane K, Cooper TB et al (1989). Serotonergic studies in patients with affective and personality disorders. Correlates with suicidal and impulsive aggressive behavior. Arch Gen Psychiatry 46: 587-599.

Cools R, Clark L, Owen AM, Robbins TW (2002). Defining the neural mechanisms of probabilistic reversal learning using event-related functional magnetic resonance imaging. J Neurosci 22: 4563-4567.

Cusack R, Brett M, Osswald K (2003). An evaluation of the use of magnetic field maps to undistort echo-planar images. NeuroImage 18: 127-142.

Dalley JW, Theobald DE, Eagle DM, Passetti F, Robbins TW (2002). Deficits in impulse control associated with tonically-elevated serotonergic function in rat prefrontal cortex. Neuropsychopharmacology 26: 716-728.

Deakin JF (1991). Depression and 5HT. Int Clin Psychopharmacol 6(Suppl 3): 23-28 discussion 29-31.

Dias R, Robbins TW, Roberts AC (1996). Dissociation in prefrontal cortex of affective and attentional shifts. Nature 380: 69-72.

Drevets WC, Price JL, Simpson Jr JR, Todd RD, Reich T, Vannier $M$ et al (1997). Subgenual prefrontal cortex abnormalities in mood disorders. Nature 386: 824-827.

Ellenbogen MA, Young SN, Dean P, Palmour RM, Benkelfat C (1996). Mood response to acute tryptophan depletion in healthy volunteers: sex differences and temporal stability. Neuropsychopharmacology 15: 465-474.

Elliott R, Rubinsztein JS, Sahakian BJ, Dolan RJ (2002). The neural basis of mood-congruent processing biases in depression. Arch Gen Psychiatry 59: 597-604.

Elliott R, Sahakian BJ, Herrod JJ, Robbins TW, Paykel ES (1997). Abnormal response to negative feedback in unipolar depression: evidence for a diagnosis specific impairment. J Neurol Neurosurg Psychiatry 63: 74-82.

Evenden JL (1999). Varieties of impulsivity. Psychopharmacology (Berl) 146: 348-361.

Fellows LK, Farah MJ (2003). Ventromedial frontal cortex mediates affective shifting in humans: evidence from a reversal learning paradigm. Brain 126: 1830-1837.

Garavan H, Ross TJ, Kaufman J, Stein EA (2003). A midline dissociation between error-processing and response-conflict monitoring. NeuroImage 20: 1132-1139.

Gehring WJ, Fencsik DE (2001). Functions of the medial frontal cortex in the processing of conflict and errors. J Neurosci 21: 9430-9437.

Graeff FG, Brandao ML, Audi EA, Schutz MT (1986). Modulation of the brain aversive system by GABAergic and serotonergic mechanisms. Behav Brain Res 22: 173-180.

Harmer CJ, Rogers RD, Tunbridge E, Cowen PJ, Goodwin GM (2003). Tryptophan depletion decreases the recognition of fear in female volunteers. Psychopharmacology (Berl) 167: 411-417.

Harrison AA, Everitt BJ, Robbins TW (1997). Central 5-HT depletion enhances impulsive responding without affecting the accuracy of attentional performance: interactions with dopaminergic mechanisms. Psychopharmacology (Berl) 133: 329-342.

Hornak J, O’Doherty J, Bramham J, Rolls ET, Morris RG, Bullock $P R$ et al (2004). Reward-related reversal learning after surgical excisions in orbito-frontal or dorsolateral prefrontal cortex in humans. J Cogn Neurosci 16: 463-478.

Iversen SD, Mishkin M (1970). Perseverative interference in monkeys following selective lesions of the inferior prefrontal convexity. Exp Brain Res 11: 376-386. 
Jones B, Mishkin M (1972). Limbic lesions and the problem of stimulus-reinforcement associations. Exp Neurol 36: 362-377.

Kerns JG, Cohen JD, MacDonald III AW, Cho RY, Stenger VA, Carter CS (2004). Anterior cingulate conflict monitoring and adjustments in control. Science 303: 1023-1026.

Koskinen T, Ruotsalainen S, Sirvio J (2000). The 5-HT(2) receptor activation enhances impulsive responding without increasing motor activity in rats. Pharmacol Biochem Behav 66: 729-738.

Kringelbach ML, Rolls ET (2003). Neural correlates of rapid reversal learning in a simple model of human social interaction. NeuroImage 20: 1371-1383.

Lacerda AL, Keshavan MS, Hardan AY, Yorbik O, Brambilla P, Sassi RB et al (2004). Anatomic evaluation of the orbitofrontal cortex in major depressive disorder. Biol Psychiatry 55: 353-358.

Leyton M, Okazawa H, Diksic M, Paris J, Rosa P, Mzengeza S et al (2001). Brain regional alpha-[11C]methyl-L-tryptophan trapping in impulsive subjects with borderline personality disorder. Am J Psychiatry 158: 775-782.

Liu YP, Wilkinson LS, Robbins TW (2004). Effects of acute and chronic buspirone on impulsive choice and efflux of 5-HT and dopamine in hippocampus, nucleus accumbens and prefrontal cortex. Psychopharmacology (Berl) 173: 175-185.

MacDonald III AW, Cohen JD, Stenger VA, Carter CS (2000). Dissociating the role of the dorsolateral prefrontal and anterior cingulate cortex in cognitive control. Science 288: 1835-1838.

Marek GJ, Aghajanian GK (1998). 5-Hydroxytryptamine-induced excitatory postsynaptic currents in neocortical layer $\mathrm{V}$ pyramidal cells: suppression by mu-opiate receptor activation. Neuroscience 86: 485-497.

Mayberg HS, Liotti M, Brannan SK, McGinnis S, Mahurin RK, Jerabek PA et al (1999). Reciprocal limbic-cortical function and negative mood: converging PET findings in depression and normal sadness. Am J Psychiatry 156: 675-682.

Merali Z, Du L, Hrdina P, Palkovits M, Faludi G, Poulter MO et al (2004). Dysregulation in the suicide brain: mRNA expression of corticotropin-releasing hormone receptors and GABA(A) receptor subunits in frontal cortical brain region. J Neurosci 24: 1478-1485.

Mobini S, Chiang TJ, Ho MY, Bradshaw CM, Szabadi E (2000). Effects of central 5-hydroxytryptamine depletion on sensitivity to delayed and probabilistic reinforcement. Psychopharmacology (Berl) 152: 390-397.

Murphy FC, Michael A, Robbins TW, Sahakian BJ (2003). Neuropsychological impairment in patients with major depressive disorder: the effects of feedback on task performance. Psychol Med 33: 455-467.

Murphy FC, Smith KA, Cowen PJ, Robbins TW, Sahakian BJ (2002). The effects of tryptophan depletion on cognitive and affective processing in healthy volunteers. Psychopharmacology (Berl) 163: 42-53.

Nishizawa S, Benkelfat C, Young SN, Leyton M, Mzengeza S, de Montigny C et al (1997). Differences between males and females in rates of serotonin synthesis in human brain. Proc Natl Acad Sci USA 94: 5308-5313.

O’Doherty J, Kringelbach ML, Rolls ET, Hornak J, Andrews C (2001). Abstract reward and punishment representations in the human orbitofrontal cortex. Nat Neurosci 4: 95-102.

Orosco M, Rouch C, Beslot F, Feurte S, Regnault A, Dauge V (2004). Alpha-lactalbumin-enriched diets enhance serotonin release and induce anxiolytic and rewarding effects in the rat. Behav Brain Res 148: 1-10.

Paus T, Petrides MJ, Evans AC, Meyer E. (1993). Role of the human anterior cingulate cortex in the control of oculomotor, manual, and speech responses: a positron emission tomography study. J Neurophysiol 70: 453-469.

Park SB, Coull JT, McShane RH, Young AH, Sahakian BJ, Robbins TW et al (1994). Tryptophan depletion in normal volunteers produces selective impairments in learning and memory. Neuropharmacology 33: 575-588.

Patkina NA, Lapin IP (1976). Effect of serotoninergic drugs on positive and negative reinforcing systems in cats. Pharmacol Biochem Behav 5: 241-245.

Poeggel G, Nowicki L, Braun K (2003). Early social deprivation alters monoaminergic afferents in the orbital prefrontal cortex of Octodon degus. Neuroscience 116: 617-620.

Preece MA, Dalley JW, Theobald DE, Robbins TW, Reynolds GP (2004). Region specific changes in forebrain 5-hydroxytryptamine1A and 5-hydroxytryptamine2A receptors in isolation-reared rats: an in vitro autoradiography study. Neuroscience 123: 725-732.

Puumala T, Sirvio J (1998). Changes in activities of dopamine and serotonin systems in the frontal cortex underlie poor choice accuracy and impulsivity of rats in an attention task. Neuroscience 83: 489-499.

Puig MV, Artigas F, Celada P (2004). Modulation of the activity of pyramidal neurons in rat prefrontal cortex by raphe stimulation in vivo: involvement of serotonin and GABA. Cereb Cortex, Epub ahead of print.

Redgrave P, Horrell RI (1976). Potentiation of central reward by localised perfusion of acetylcholine and 5-hydroxytryptamine. Nature 262: 305-307.

Riedel WJ, Klaassen T, Deutz NE, van Someren A, van Praag HM (1999). Tryptophan depletion in normal volunteers produces selective impairment in memory consolidation. Psychopharmacology (Berl) 141: 362-369.

Robbins TW (2000). Chemical neuromodulation of frontalexecutive functions in humans and other animals. Exp Brain Res 133: 130-138.

Rogers RD, Everitt BJ, Baldacchino A, Blackshaw AJ, Swainson R, Wynne $\mathrm{K}$ et al (1999). Dissociable deficits in the decisionmaking cognition of chronic amphetamine abusers, opiate abusers, patients with focal damage to prefrontal cortex, and tryptophan-depleted normal volunteers: evidence for monoaminergic mechanisms. Neuropsychopharmacology 20: 322-339.

Rogers RD, Tunbridge EM, Bhagwagar Z, Drevets WC, Sahakian BJ, Carter CS (2003). Tryptophan depletion alters the decisionmaking of healthy volunteers through altered processing of reward cues. Neuropsychopharmacology 28: 153-162.

Rubinsztein JS, Fletcher PC, Rogers RD, Ho LW, Aigbirhio FI, Paykel ES et al (2001). Decision-making in mania: a PET study. Brain 124: 2550-2563.

Sasaki-Adams DM, Kelley AE (2001). Serotonin-dopamine interactions in the control of conditioned reinforcement and motor behavior. Neuropsychopharmacology 25: 440-452.

Smith KA, Fairburn CG, Cowen PJ (1997). Relapse of depression after rapid depletion of tryptophan. Lancet 349: 915-919.

Smith RL, Kennedy CH (2003). Increases in avoidance responding produced by REM sleep deprivation or serotonin depletion are reversed by administration of 5-hydroxytryptophan. Behav Brain Res 140: 81-86.

Smith SM (2002). Fast robust automated brain extraction. Hum Brain Mapp 17: 143-155.

Soubrie P (1986). Reconciling the role of central serotonin neurons in human and animal behaviour. Behav Brain Res 9: 319-364.

Steffens DC, Wagner HR, Levy RM, Horn KA, Krishnan KR (2001). Performance feedback deficit in geriatric depression. Biol Psychiatry 50: 358-363.

Swainson R, Rogers RD, Sahakian BJ, Summers BA, Polkey CE, Robbins TW (2000). Probabilistic learning and reversal deficits in patients with Parkinson's disease or frontal or temporal lobe lesions: possible adverse effects of dopaminergic medication. Neuropsychologia 38: 596-612.

Tzourio-Mazoyer N, Landeau B, Papathanassiou D, Crivello F, Etard O, Delcroix N et al (2002). Automated anatomical labeling of activations in SPM using a macroscopic anatomical parcellation of the MNI MRI single-subject brain. Neuroimage 15: 273-289. 
Walderhaug E, Lunde H, Nordvik JE, Landro NI, Refsum H, Magnusson A (2002). Lowering of serotonin by rapid tryptophan depletion increases impulsiveness in normal individuals. Psychopharmacology (Berl) 164: 385-391.

Watson D, Clark LA, Tellegen A (1988). Development and validation of brief measures of positive and negative affect: the PANAS scales. J Pers Soc Psychol 54: 1063-1070.

Wilkinson LS, Humby T, Robbins TW, Everitt BJ (1995). Differential effects of forebrain 5-hydroxytryptamine depletions on Pavlovian aversive conditioning to discrete and contextual stimuli in the rat. Eur J Neurosci 7: 2042-2052.

Williams WA, Shoaf SE, Hommer D, Rawlings R, Linnoila M (1999). Effects of acute tryptophan depletion on plasma and cerebrospinal fluid tryptophan and 5-hydroxyindoleacetic acid in normal volunteers. J Neurochem 72: 1641-1647.

Young SN, Smith SE, Pihl RO, Ervin FR (1985). Tryptophan depletion causes a rapid lowering of mood in normal males. Psychopharmacology (Berl) 87: 173-177. 